Supplementary information for the paper:

J. Phys. Chem. (A) 2008

\title{
Structure-Energy Relationships in Unsaturated Esters of Carboxylic Acids. Thermochemical Measurements and Ab Initio Calculations
}

Vladimir N. Emel'yanenko, Alexey V. Toktonov, Svetlana A. Kozlova, Sergey P. Verevkin, ${ }^{a}$ Department of Physical Chemistry, University of Rostock, 18051 Rostock, Germany

Vasyl Andrushko, Natalia Andrushko, Armin Börner

Leibniz Institut für Katalyse an der Universität Rostock e.V., Albert-Einstein Str. 29a, 18059 Rostock, Germany

Spectroscopic data for alkyl 3-methylbut-2-enoates:

\section{i-Propyl 3-methylbut-2-enoate}<smiles>CC(C)=CC(=O)OC(C)C</smiles>

b.p. $98-100^{\circ} \mathrm{C}(7.5 \mathrm{mbar})$.

${ }^{1} \mathrm{H}$ NMR (300.13 MHz, $\left.\mathrm{CDCl}_{3}\right), \delta: 1.25\left(\mathrm{~d}, J=6.30 \mathrm{~Hz}, 3 \mathrm{H}, \mathrm{O}-\mathrm{CH}\left(\mathrm{CH}_{3}\right)_{2}\right), 1.88(\mathrm{~d}, J=1.34 \mathrm{~Hz}$, $\left.3 \mathrm{H}, \mathrm{HC}=\mathrm{CCH}_{3} \mathrm{CH}_{3}\right), 2.16\left(\mathrm{~d}, J=1.34 \mathrm{~Hz}, 3 \mathrm{H}, \mathrm{HC}=\mathrm{CCH}_{3} \mathrm{CH}_{3}\right), 5.03$ (sept, $J=6.36 \mathrm{~Hz}, 1 \mathrm{H}, \mathrm{O}-$ $\left.\mathrm{C} H\left(\mathrm{CH}_{3}\right)_{2}\right) ; 5.65$ (sept, $J=1.35 \mathrm{~Hz}, 1 \mathrm{H},\left(\mathrm{CH}_{3}\right)_{2} \mathrm{C}=\mathrm{CH}$ );

${ }^{13} \mathrm{C}$ NMR (75.477 MHz, $\left.\mathrm{CDCl}_{3}\right), \delta: 20.13\left(\mathrm{CH}_{3} \mathrm{C}\right), 22.00\left(\left(\mathrm{CH}_{3}\right)_{2} \mathrm{CH}\right), 27.37\left(\mathrm{CH}_{3} \mathrm{C}\right), 66.55$ $\left.\left(\left(\mathrm{CH}_{3}\right)_{2} \mathrm{CH}\right), 116.64\left(\mathrm{CH}_{3}\right)_{2} \mathrm{C}=\mathrm{CH}\right), 155.95(\mathrm{CH}-\mathrm{C}), 166.31(\mathrm{CO})$;

MS (EI, $\mathrm{C}_{8} \mathrm{H}_{14} \mathrm{O}_{2}, \mathrm{M}=142.2 \mathrm{~g} / \mathrm{mol}$ ) m/z = 142 (6), 101 (11), 100 (100), 85 (29), 84 (9), 83 (98), 82 (44), 59 (11), 55 (32), 43 (25), 41 (15), 39 (23), 29 (11).

\section{Structural isomer: i-Propyl 3-methylbut-3-enoate}<smiles>C=C(C)CC(=O)OC(C)C</smiles>

MS (EI, $\left.\mathrm{C}_{8} \mathrm{H}_{14} \mathrm{O}_{2}, \mathrm{M}=142.2 \mathrm{~g} / \mathrm{mol}\right) \mathrm{m} / \mathrm{z}=142$ (4), 101 (4), 100 (62), 85 (2), 84 (2), 83 (37), 82 (6), 72 (18), 59 (3), 58 (5), 57 (7), 56 (10), 55 (54), 54 (6), 53 (6), 43 (100), 42 (6), 41 (31), 39 (21), 29 $(10)$. 
<smiles>CCCOC(=O)C=C(C)C</smiles>

b.p. $100-102^{\circ} \mathrm{C}(0.5 \mathrm{mbar})$.

${ }^{1} \mathrm{H}$ NMR (300.13 MHz, $\left.\mathrm{CDCl}_{3}\right), \delta: 0.954\left(\mathrm{t}, J=7.42 \mathrm{~Hz}, 3 \mathrm{H}, \mathrm{O}-\left(\mathrm{CH}_{2}\right)_{2}-\mathrm{CH}_{3}\right), 1.603-1.730(\mathrm{~m}, 2 \mathrm{H}$, $\left.\mathrm{O}-\mathrm{CH}_{2}-\mathrm{CH}_{2}-\mathrm{CH}_{3}\right), 1.893\left(\mathrm{~d}, J=1.34 \mathrm{~Hz}, 3 \mathrm{H}, \mathrm{HC}=\mathrm{CCH}_{3} \mathrm{CH}_{3}\right), 2.168$ (d, $J=1.34 \mathrm{~Hz}, 3 \mathrm{H}$, $\left.\mathrm{HC}=\mathrm{CCH}_{3} \mathrm{CH}_{3}\right), 4.046\left(\mathrm{t}, J=6.74 \mathrm{~Hz}, 2 \mathrm{H}, \mathrm{O}-\mathrm{CH}_{2}\right), 5.684\left(\mathrm{sept}, J=1.34 \mathrm{~Hz}, 1 \mathrm{H},\left(\mathrm{CH}_{3}\right)_{2} \mathrm{C}=\mathrm{CH}\right)$;

${ }^{13} \mathrm{C}$ NMR (75.477 MHz, $\left.\mathrm{CDCl}_{3}\right), \delta: 10.48\left(\mathrm{CH}_{3}\right), 20.15\left(\mathrm{CH}_{3}\right), 22.09\left(\mathrm{CH}_{2}\right), 27.36\left(\mathrm{CH}_{3}\right), 64.14$ $\left(\mathrm{CH}_{2}\right), 116.16(\mathrm{CH}), 156.29(\mathrm{C}), 166.85(\mathrm{C})$.

MS (EI, $\left.\mathrm{C}_{8} \mathrm{H}_{14} \mathrm{O}_{2}, \mathrm{M}=142.2 \mathrm{~g} / \mathrm{mol}\right) \mathrm{m} / \mathrm{z}=142$ (18), 101 (14), 100 (64), 85 (21), 84 (7), 83 (100), 82 (34), 55 (32), 54 (5), 53 (9), 43 (11), 41 (11), 40 (3), 39 (19), 29 (13).

\section{Structural isomer: n-Propyl 3-methylbut-3-enoate}<smiles>C=C(C)CC(=O)OCCC</smiles>

${ }^{1} \mathrm{H}$ NMR (300.13 MHz, $\left.\mathrm{CDCl}_{3}\right), \delta: 0.946\left(\mathrm{t}, J=7.42 \mathrm{~Hz}, 3 \mathrm{H}, \mathrm{O}-\left(\mathrm{CH}_{2}\right)_{3}-\mathrm{CH}_{3}\right)$, 1.6-1.7 (m, 2H, O$\left.\mathrm{CH}_{2}-\mathrm{CH}_{2}-\mathrm{CH}_{3}\right), 1.818\left(\mathrm{~s}, 3 \mathrm{H}, \mathrm{H}_{2} \mathrm{C}=\mathrm{C}-\mathrm{CH}_{3}\right), 3.035$ (s, 1H, $\left.\mathrm{CH}_{\mathrm{a}} \mathrm{H}_{\mathrm{b}}-\mathrm{CO}_{2} \mathrm{Pr}\right), 3.039\left(\mathrm{~s}, 1 \mathrm{H}, \mathrm{CH}_{\mathrm{a}} H_{\mathrm{b}}-\right.$ $\left.\mathrm{CO}_{2} \mathrm{Pr}\right), 4.836-4.866\left(\mathrm{~m}, 1 \mathrm{H}, \mathrm{PrO}_{2} \mathrm{C}-\mathrm{C}=\mathrm{CH}_{\mathrm{a}} \mathrm{H}_{\mathrm{b}}\right), 4.894-4.924\left(\mathrm{~m}, 1 \mathrm{H}, \operatorname{PrO}_{2} \mathrm{C}-\mathrm{C}=\mathrm{CH}_{\mathrm{a}} H_{\mathrm{b}}\right)$;

MS (EI, $\mathrm{C}_{8} \mathrm{H}_{14} \mathrm{O}_{2}, \mathrm{M}=142.2 \mathrm{~g} / \mathrm{mol}$ ) m/z = 142 (27), 100 (51), 99 (3), 85 (4), 83 (50), 82 (14), 72 (37), 59 (3), 58 (9), 57 (13), 56 (25), 55 (68), 54 (12), 53 (10), 44 (4), 43 (100), 42 (9), 41 (51), 40 (7), 39 (30), 29 (19).

\section{n-Butyl 3-methylbut-2-enoate}<smiles>CCCCOC(=O)C=C(C)C</smiles>

b.p. $116-118^{\circ} \mathrm{C}(4.5 \mathrm{mbar})$.

${ }^{1} \mathrm{H}$ NMR (300.13 MHz, $\left.\mathrm{CDCl}_{3}\right), \delta: 0.937$ (t, $\left.J=7.3 \mathrm{~Hz}, 3 \mathrm{H}, \mathrm{O}-\left(\mathrm{CH}_{2}\right)_{3}-\mathrm{CH}_{3}\right), 1.32-1.46(\mathrm{~m}, 2 \mathrm{H}, \mathrm{O}-$ $\left.\left(\mathrm{CH}_{2}\right)_{2}-\mathrm{CH}_{2}-\mathrm{CH}_{3}\right), \quad 1.57-1.68\left(\mathrm{~m}, 2 \mathrm{H}, \mathrm{O}-\mathrm{CH}_{2}-\mathrm{CH}_{2}-\mathrm{CH}_{2}-\mathrm{CH}_{3}\right), 1.889$ (d, J = $1.34 \mathrm{~Hz}, 3 \mathrm{H}$, $\left.\mathrm{HC}=\mathrm{CCH}_{3} \mathrm{CH}_{3}\right), 2.166\left(\mathrm{~d}, J=1.34 \mathrm{~Hz}, 3 \mathrm{H}, \mathrm{HC}=\mathrm{CCH}_{3} \mathrm{CH}_{3}\right), 4.088\left(\mathrm{t}, J=6.67 \mathrm{~Hz}, 2 \mathrm{H}, \mathrm{O}-\mathrm{CH}_{2}\right)$, 5.676 (sept, $\left.J=1.34 \mathrm{~Hz}, 1 \mathrm{H},\left(\mathrm{CH}_{3}\right)_{2} \mathrm{C}=\mathrm{CH}\right)$;

${ }^{13} \mathrm{C}$ NMR (75.477 MHz, $\left.\mathrm{CDCl}_{3}\right), \delta: 13.72\left(\mathrm{CH}_{3}\right), 19.23\left(\mathrm{CH}_{2}\right), 20.13\left(\mathrm{CH}_{3}\right), 27.34\left(\mathrm{CH}_{3}\right), 30.81$ $\left(\mathrm{CH}_{2}\right), 64.51\left(\mathrm{CH}_{2}\right), 116.16(\mathrm{CH}), 156.26(\mathrm{C}), 166.83(\mathrm{C})$;

MS (EI, $\left.\mathrm{C}_{9} \mathrm{H}_{16} \mathrm{O}_{2}, \mathrm{M}=156.22 \mathrm{~g} / \mathrm{mol}\right) \mathrm{m} / \mathrm{z}=156$ (11), 101 (23), 100 (65), 85 (14), 84 (6), 83 (100), 82 (24), 57 (7), 56 (6), 55 (27), 53 (7), 41 (12), 39 (14), 29 (14). 


\section{Structural isomer: n-Butyl 3-methylbut-3-enoate}<smiles>C=C(C)CC(=O)OCCCC</smiles>

${ }^{1} \mathrm{H}$ NMR (300.13 MHz, $\mathrm{CDCl}_{3}$ ), $\delta: 0.937$ (t, $\left.J=7.3 \mathrm{~Hz}, 3 \mathrm{H}, \mathrm{O}-\left(\mathrm{CH}_{2}\right)_{3}-\mathrm{CH}_{3}\right), 1.32-1.46$ (m, 2H, O$\left.\left(\mathrm{CH}_{2}\right)_{2}-\mathrm{CH}_{2}-\mathrm{CH}_{3}\right), 1.57-1.68\left(\mathrm{~m}, 2 \mathrm{H}, \mathrm{O}-\mathrm{CH}_{2}-\mathrm{CH}_{2}-\mathrm{CH}_{2}-\mathrm{CH}_{3}\right), 1.814$ (s, 3H, $\left.\mathrm{H}_{2} \mathrm{C}=\mathrm{C}-\mathrm{CH}_{3}\right), 3.029$ (s, $\left.1 \mathrm{H}, \mathrm{CH}_{\mathrm{a}} \mathrm{H}_{\mathrm{b}}-\mathrm{CO}_{2} \mathrm{Bu}\right), 3.032\left(\mathrm{~s}, 1 \mathrm{H}, \mathrm{CH}_{\mathrm{a}} \mathrm{H}_{\mathrm{b}}-\mathrm{CO}_{2} \mathrm{Bu}\right), 4.832-4.863\left(\mathrm{~m}, 1 \mathrm{H}, \mathrm{BuO}_{2} \mathrm{C}-\mathrm{C}=\mathrm{CH}_{\mathrm{a}} \mathrm{H}_{\mathrm{b}}\right), 4.892-$ $4.923\left(\mathrm{~m}, 1 \mathrm{H}, \mathrm{BuO}_{2} \mathrm{C}-\mathrm{C}=\mathrm{CH}_{\mathrm{a}} H_{\mathrm{b}}\right)$;

${ }^{13} \mathrm{C}$ NMR (75.477 MHz, $\left.\mathrm{CDCl}_{3}\right), \delta: 13.72\left(\mathrm{CH}_{3}\right), 19.20\left(\mathrm{CH}_{2}\right), 22.43\left(\mathrm{CH}_{2}\right), 30.65\left(\mathrm{CH}_{3}\right), 43.49$ $\left(\mathrm{CH}_{2}\right), 64.47\left(\mathrm{CH}_{2}\right), 114.51\left(\mathrm{CH}_{2}\right), 138.7(C), 171.5(C)$;

MS (EI, $\mathrm{C}_{9} \mathrm{H}_{16} \mathrm{O}_{2}, \mathrm{M}=156.22 \mathrm{~g} / \mathrm{mol}$ ) m/z = 156 (20), 101 (15), 100 (95), 99 (5), 96 (7), 85 (8), 84 (4), 83 (70), 82 (21), 72 (64), 59 (9), 58 (13), 57 (96), 56 (39), 55 (91), 54 (13), 53 (14), 43 (15), 42 (9), 41 (100), 40 (8), 39 (40), 29 (73).

TABLE S1. Results for typical combustion experiments at $\mathrm{T}=298.15 \mathrm{~K}\left(\mathrm{p}^{\circ}=0.1 \mathrm{MPa}\right)$ of the Methyl 3-methylbut-2-enoate. ${ }^{a}$

\begin{tabular}{ccccccc}
\hline $\mathrm{m}$ (substance) $/ \mathrm{g} \mathrm{b}$ & 0.379873 & 0.343892 & 0.33505 & 0.319133 & 0.354138 & 0.342581 \\
$\mathrm{~m}^{\prime}($ cotton $) / \mathrm{g} \mathrm{b}$ & 0.00113 & 0.001097 & 0.001111 & 0.000995 & 0.000965 & 0.001204 \\
$\mathrm{~m}^{\prime \prime}($ polythen $) / \mathrm{g} \mathrm{b}$ & 0.425501 & 0.396752 & 0.365043 & 0.356817 & 0.358857 & 0.400988 \\
$\Delta \mathrm{T}_{\mathrm{c}} / \mathrm{K}^{\mathrm{c}}$ & 2.09231 & 1.93113 & 1.8139 & 1.75629 & 1.83251 & 1.94145 \\
$\left(\varepsilon_{\mathrm{calor}}\right) \cdot\left(-\Delta \mathrm{T}_{\mathrm{c}}\right) / \mathrm{J}$ & -30981 & -28594.5 & -26858.6 & -26005.6 & -27134.2 & -28747.3 \\
$\left(\varepsilon_{\mathrm{cont}}\right) \cdot\left(-\Delta \mathrm{T}_{\mathrm{c}}\right) / \mathrm{J}$ & -39.96 & -36.51 & -33.89 & -32.7 & -34.28 & -36.69 \\
$\Delta \mathrm{U}_{\mathrm{decomp}} \mathrm{HNO}_{3} / \mathrm{J}$ & 58.53 & 53.75 & 50.47 & 49.57 & 50.77 & 54.35 \\
$\Delta \mathrm{U}_{\mathrm{corr}} / \mathrm{J}^{\mathrm{d}}$ & 10.94 & 9.92 & 9.23 & 8.87 & 9.4 & 9.97 \\
$-\mathrm{m}^{\prime} \cdot \Delta_{\mathrm{c}} \mathrm{u}^{\prime} / \mathrm{J}$ & 19.15 & 18.59 & 18.83 & 16.86 & 16.35 & 20.4 \\
$-\mathrm{m}^{\prime \prime} \cdot \Delta_{\mathrm{c}} \mathrm{u}^{\prime \prime} / \mathrm{J}$ & 19726.65 & 18393.82 & 16923.76 & 16542.39 & 16636.97 & 18590.2 \\
$\Delta_{\mathrm{c}} \mathrm{u}^{\circ}\left(\right.$ liq) $/\left(\mathrm{J} \cdot \mathrm{g}^{-1}\right)$ & -29498.5 & -29529.4 & -29518.4 & -29519.5 & -29522.4 & -29508.6
\end{tabular}

a For the definition of the symbols see reference 18 , macrocalorimeter: $\mathrm{T}_{\mathrm{h}}=298.15 \mathrm{~K}$; V(bomb) $=$ $0.2664 \mathrm{dm}^{3} ; \mathrm{p}^{\mathrm{i}}($ gas $)=3.04 \mathrm{MPa} ; \mathrm{m}^{\mathrm{i}}\left(\mathrm{H}_{2} \mathrm{O}\right)=1.00 \mathrm{~g} ;{ }^{\mathrm{b}}$ Masses obtained from apparent masses. ${ }^{\mathrm{c}} \Delta \mathrm{T}_{\mathrm{c}}$ $=\mathrm{T}^{\mathrm{f}}-\mathrm{T}^{\mathrm{i}}+\Delta \mathrm{T}_{\text {corr }} ;\left(\varepsilon_{\text {cont }}\right) \cdot\left(-\Delta \mathrm{T}_{\mathrm{c}}\right)=\left(\varepsilon_{\text {cont }}^{\mathrm{i}}\right) \cdot\left(\mathrm{T}^{\mathrm{i}}-298.15 \mathrm{~K}\right)+\left(\varepsilon_{\text {cont }}^{\mathrm{f}}\right) \cdot\left(298.15 \mathrm{~K}-\mathrm{T}^{\mathrm{f}}+\Delta \mathrm{T}_{\text {corr. }}\right) \cdot{ }^{\mathrm{d}}$ $\Delta \mathrm{U}_{\text {corr }}$, the correction to standard states, is the sum of items 81 to 85,87 to 90,93 , and 94 in reference $18 .{ }^{\mathrm{f}} \varepsilon=14807.1 \mathrm{~J} \cdot \mathrm{K}^{-1}$ 
TABLE S2. Results for typical combustion experiments at $\mathrm{T}=298.15 \mathrm{~K}\left(\mathrm{p}^{\circ}=0.1 \mathrm{MPa}\right)$ of the Ethyl 3-methylbut-2-enoate.

\begin{tabular}{|c|c|c|c|c|c|c|c|c|}
\hline $\mathrm{m}$ (substance) $/ \mathrm{g} b$ & 0.504222 & 0.389369 & 0.473725 & 0.456115 & 0.413918 & 0.440839 & 0.4336 & 0.326432 \\
\hline $\mathrm{m}^{\prime}($ cotton $) / \mathrm{g} b$ & 0.002959 & 0.002853 & 0.002824 & 0.003095 & 0.003157 & 0.003516 & 0.003215 & 0.002931 \\
\hline m"(polythen) /g b & 0.288531 & 0.279383 & 0.289855 & 0.305124 & 0.288676 & 0.297724 & 0.28195 & 0.29275 \\
\hline$\Delta \mathrm{T}_{\mathrm{c}} / \mathrm{K}^{\mathrm{c}}$ & 1.97317 & 1.70302 & 1.91422 & 1.92471 & 1.78295 & 1.86872 & 1.80439 & 1.61157 \\
\hline$\left(\varepsilon_{\text {calor }}\right) \cdot\left(-\Delta \mathrm{T}_{\mathrm{c}}\right) / \mathrm{J}$ & -29216.9 & -25216.7 & -28344.1 & -28499.4 & -26400.4 & -27670.4 & -26717.8 & -23862.6 \\
\hline$\left(\varepsilon_{\text {cont }}\right) \cdot\left(-\Delta \mathrm{T}_{\mathrm{c}}\right) / \mathrm{J}$ & -37.48 & -31.52 & -36.21 & -36.37 & -33.35 & -35.09 & -33.74 & -29.68 \\
\hline$\Delta \mathrm{U}_{\text {decomp }} \mathrm{HNO}_{3} / \mathrm{J}$ & 54.95 & 48.98 & 54.95 & 52.26 & 49.57 & 53.75 & 49.57 & 46.89 \\
\hline$\Delta \mathrm{U}_{\text {corr }} / \mathrm{J}^{\mathrm{d}}$ & 10.39 & 8.55 & 9.93 & 9.98 & 9.09 & 9.6 & 9.25 & 7.91 \\
\hline$-\mathrm{m}^{\prime} \cdot \Delta_{\mathrm{c}} \mathrm{u}^{\prime} / \mathrm{J}$ & 50.14 & 48.34 & 47.85 & 52.44 & 53.5 & 59.58 & 54.48 & 49.67 \\
\hline$-\mathrm{m}^{\prime \prime} \cdot \Delta_{\mathrm{c}} \mathrm{u}^{\prime \prime} / \mathrm{J}$ & 13376.59 & 12952.48 & 13437.97 & 14145.85 & 13383.31 & 13802.78 & 13071.48 & 13572.18 \\
\hline$\Delta_{\mathrm{c}} \mathrm{u}^{\circ}$ (liq) $/\left(\mathrm{J} \cdot \mathrm{g}^{-1}\right)$ & -31260.7 & -31306.8 & -31304.2 & -31297.5 & -31258 & -31257.9 & -31288.7 & -31295 \\
\hline
\end{tabular}

TABLE S3. Results for typical combustion experiments at $\mathrm{T}=298.15 \mathrm{~K}\left(\mathrm{p}^{\circ}=0.1 \mathrm{MPa}\right)$ of the Propyl 3-methylbut-2-enoate.

\begin{tabular}{cccccc}
\hline $\mathrm{m}$ (substance) $/ \mathrm{g} b$ & 0.411326 & 0.427197 & 0.426025 & 0.441383 & 0.376785 \\
$\mathrm{~m}^{\prime}($ cotton $) / \mathrm{g} \mathrm{b}$ & 0.001181 & 0.001122 & 0.001156 & 0.001182 & 0.001161 \\
$\mathrm{~m}^{\prime \prime}($ polythen $) / \mathrm{g} \mathrm{b}$ & 0.290363 & 0.28717 & 0.28544 & 0.300262 & 0.275804 \\
$\Delta \mathrm{T}_{\mathrm{c}} / \mathrm{K}^{\mathrm{c}}$ & 1.82339 & 1.84823 & 1.84082 & 1.9202 & 1.70053 \\
$\left(\varepsilon_{\mathrm{calor}}\right) \cdot\left(-\Delta \mathrm{T}_{\mathrm{c}}\right) / \mathrm{J}$ & -26999.2 & -27367 & -27257.2 & -28432.7 & -25180 \\
$\left(\varepsilon_{\mathrm{cont}}\right) \cdot\left(-\Delta \mathrm{T}_{\mathrm{c}}\right) / \mathrm{J}$ & -34.24 & -34.7 & -34.54 & -36.32 & -31.54 \\
$\Delta \mathrm{U}_{\mathrm{decomp}} \mathrm{HNO}_{3} / \mathrm{J}$ & 45.39 & 50.77 & 50.47 & 53.16 & 45.39 \\
$\Delta \mathrm{U}_{\mathrm{corr}} / \mathrm{Jd}$ & 9.08 & 9.19 & 9.14 & 9.64 & 8.31 \\
$-\mathrm{m}^{\prime} \cdot \Delta_{\mathrm{c}} \mathrm{u}^{\prime} / \mathrm{J}$ & 20.01 & 19.01 & 19.59 & 20.03 & 19.67 \\
$-\mathrm{m}^{\prime \prime} \cdot \Delta_{\mathrm{c}} \mathrm{u}^{\prime \prime} / \mathrm{J}$ & 13461.52 & 13313.49 & 13233.28 & 13920.45 & 12786.55 \\
$\Delta_{\mathrm{c}} \mathrm{u}^{\circ}(\mathrm{liq}) /(\mathrm{J} \cdot \mathrm{g}-1)$ & -32814.4 & -32793.3 & -32813.2 & -32773.6 & -32781.5 \\
\hline
\end{tabular}


TABLE S4. Results for typical combustion experiments at $\mathrm{T}=298.15 \mathrm{~K}\left(\mathrm{p}^{\circ}=0.1 \mathrm{MPa}\right)$ of the iso-Propyl 3-methylbut-2-enoate.

\begin{tabular}{ccccccc}
$\mathrm{m}$ (substance) $/ \mathrm{g} b$ & 0.420622 & 0.366099 & 0.343037 & 0.426375 & 0.383103 & 0.397856 \\
$\mathrm{~m}^{\prime}($ cotton $) / \mathrm{g} b$ & 0.001203 & 0.001291 & 0.001265 & 0.001014 & 0.001133 & 0.001296 \\
$\mathrm{~m}^{\prime \prime}($ polythen $) / \mathrm{g} \mathrm{b}$ & 0.293636 & 0.303801 & 0.292052 & 0.325973 & 0.321762 & 0.335523 \\
$\Delta \mathrm{T}_{\mathrm{c}} / \mathrm{K}^{\mathrm{c}}$ & 1.85259 & 1.76241 & 1.67478 & 1.96624 & 1.85715 & 1.93265 \\
$\left(\varepsilon_{\mathrm{calor}}\right) \cdot\left(-\Delta \mathrm{T}_{\mathrm{c}}\right) / \mathrm{J}$ & -27431.4 & -26096.2 & -24798.6 & -29114.4 & -27499 & -28616.9 \\
$\left(\varepsilon_{\mathrm{cont}}\right) \cdot\left(-\Delta \mathrm{T}_{\mathrm{c}}\right) / \mathrm{J}$ & -34.77 & -32.78 & -30.92 & -37.3 & -34.92 & -36.49 \\
$\Delta \mathrm{U}_{\mathrm{decomp}} \mathrm{HNO}_{3} / \mathrm{J}$ & 52.26 & 50.77 & 48.68 & 55.55 & 51.36 & 55.55 \\
$\Delta \mathrm{U}_{\mathrm{corr}} / \mathrm{J}$ & 9.2 & 8.59 & 8.08 & 9.85 & 9.16 & 9.58 \\
$-\mathrm{m}^{\prime} \cdot \Delta_{\mathrm{c}} \mathrm{u}^{\prime} / \mathrm{J}$ & 20.38 & 21.88 & 21.44 & 17.18 & 19.2 & 21.96 \\
$-\mathrm{m}^{\prime \prime} \cdot \Delta_{\mathrm{c}} \mathrm{u}^{\prime \prime} / \mathrm{J}$ & 13613.26 & 14084.52 & 13539.82 & 15112.43 & 14917.21 & 15555.18 \\
$\Delta_{\mathrm{c}} \mathrm{u}^{\circ}(\mathrm{liq}) /(\mathrm{J} \cdot \mathrm{g}-1)$ & -32739.9 & -32677.6 & -32683 & -32733.3 & -32724.8 & -32703.1 \\
\hline
\end{tabular}

TABLE S5. Results for typical combustion experiments at $\mathrm{T}=298.15 \mathrm{~K}\left(\mathrm{p}^{\circ}=0.1 \mathrm{MPa}\right)$ of the Butyl 3-methylbut-2-enoate.

\begin{tabular}{ccccccc}
$\mathrm{m}$ (substance) $/ \mathrm{g} b$ & 0.369233 & 0.356069 & 0.377629 & 0.387514 & 0.395691 & 0.35939 \\
$\mathrm{~m}^{\prime}($ cotton $) / \mathrm{g} \mathrm{b}$ & 0.001205 & 0.001407 & 0.001315 & 0.001386 & 0.001099 & 0.001196 \\
$\mathrm{~m}^{\prime \prime}($ polythen $) / \mathrm{g} \mathrm{b}$ & 0.283264 & 0.290332 & 0.275982 & 0.28562 & 0.289821 & 0.285788 \\
$\Delta \mathrm{T}_{\mathrm{c}} / \mathrm{K}^{\mathrm{c}}$ & 1.73734 & 1.72999 & 1.73389 & 1.78746 & 1.81983 & 1.72347 \\
$\left(\varepsilon_{\text {calor }}\right) \cdot\left(-\Delta \mathrm{T}_{\mathrm{c}}\right) / \mathrm{J}$ & -25725 & -25616.1 & -25673.9 & -26467.1 & -26946.5 & -25519.7 \\
$\left(\varepsilon_{\mathrm{cont}}\right) \cdot\left(-\Delta \mathrm{T}_{\mathrm{c}}\right) / \mathrm{J}$ & -32.28 & -32.14 & -32.23 & -33.35 & -34.03 & -31.96 \\
$\Delta \mathrm{U}_{\mathrm{decomp}} \mathrm{HNO}_{3} / \mathrm{J}$ & 48.68 & 48.98 & 49.57 & 52.56 & 52.56 & 49.87 \\
$\Delta \mathrm{U}_{\mathrm{corr}} / \mathrm{Jd}$ & 8.31 & 8.24 & 8.3 & 8.58 & 8.76 & 8.18 \\
$-\mathrm{m}^{\prime} \cdot \Delta_{\mathrm{c}} \mathrm{u}^{\prime} / \mathrm{J}$ & 20.42 & 23.84 & 22.28 & 23.49 & 18.62 & 20.27 \\
$-\mathrm{m}^{\prime \prime} \cdot \Delta_{\mathrm{c}} \mathrm{u}^{\prime \prime} / \mathrm{J}$ & 13132.4 & 13460.08 & 12794.8 & 13241.63 & 13436.39 & 13249.42 \\
$\Delta_{\mathrm{c}} \mathrm{u}^{\circ}\left(\right.$ liq) $/\left(\mathrm{J} \cdot \mathrm{g}^{-1}\right)$ & -33982.6 & -34002.2 & -33978.4 & -33996.8 & -34026.9 & -34012.8 \\
\hline
\end{tabular}


Table S6. Total energies at $0 \mathrm{~K}$ and enthalpies at 298.15 $\mathrm{K}$ (in Hartree) of the molecules studied in this work.

\begin{tabular}{|c|c|c|c|c|}
\hline Compounds & $\mathrm{E}_{0}$ & $\mathrm{ZPE}^{\mathrm{a}}$ & $\mathrm{TCH}$ & $\mathrm{H}_{298} \mathrm{G} 3 \mathrm{MP} 2$ \\
\hline cis- $\mathrm{CH}_{3}-\mathrm{CH}=\mathrm{CH}-\mathrm{CH}_{3}$ & -157.224768 & 0.108673 & 0.115100 & -156.902105 \\
\hline trans- $\mathrm{CH}_{3}-\mathrm{CH}=\mathrm{CH}-\mathrm{CH}_{3}$ & -157.226912 & 0.108490 & 0.114910 & -156.904036 \\
\hline cis- $\mathrm{CH}_{3}-\mathrm{CH}=\mathrm{CH}-\mathrm{CH}_{2}-\mathrm{CH}_{3}$ & -196.538122 & 0.137641 & 0.145251 & -196.134541 \\
\hline trans- $\mathrm{CH}_{3}-\mathrm{CH}=\mathrm{CH}-\mathrm{CH}_{2}-\mathrm{CH}_{3}$ & -196.540442 & 0.137382 & 0.145030 & -196.136537 \\
\hline cis- $\mathrm{CH}_{3}-\mathrm{CH}_{2}-\mathrm{CH}=\mathrm{CH}-\mathrm{CH}_{2}-\mathrm{CH}_{3}$ & -235.851484 & 0.166594 & 0.175411 & -235.367055 \\
\hline trans- $\mathrm{CH}_{3}-\mathrm{CH}_{2}-\mathrm{CH}=\mathrm{CH}-\mathrm{CH}_{2}-\mathrm{CH}_{3}$ & -235.853957 & 0.166187 & 0.175115 & -235.369082 \\
\hline cis- $\mathrm{NC}-\mathrm{CH}=\mathrm{CH}-\mathrm{CH}_{3}$ & -210.154019 & 0.079567 & 0.085973 & -209.798729 \\
\hline trans-NC-CH $=\mathrm{CH}-\mathrm{CH}_{3}$ & -210.153883 & 0.079347 & 0.085876 & -209.798111 \\
\hline cis-NC-CH=CH-CH${ }_{2}-\mathrm{CH}_{3}$ & -249.467462 & 0.108481 & 0.116142 & -249.031140 \\
\hline trans-NC-CH$=\mathrm{CH}-\mathrm{CH}_{2}-\mathrm{CH}_{3}$ & -249.467451 & 0.108234 & 0.116019 & -249.030791 \\
\hline cis-HOOC-CH=CH-CH${ }_{3}$ & -306.480389 & 0.096083 & 0.103502 & -306.032740 \\
\hline trans-HOOC-CH$=\mathrm{CH}-\mathrm{CH}_{3}$ & -306.483082 & 0.095923 & 0.103354 & -306.035550 \\
\hline cis-HOOC-CH $=\mathrm{CH}-\mathrm{CH}_{2}-\mathrm{CH}_{3}$ & -345.793677 & 0.125012 & 0.133688 & -345.265429 \\
\hline trans-HOOC-CH$=\mathrm{CH}-\mathrm{CH}_{2}-\mathrm{CH}_{3}$ & -345.796112 & 0.124827 & 0.133462 & -345.268210 \\
\hline cis- $\mathrm{HC}_{3} \mathrm{OOC}-\mathrm{CH}=\mathrm{CH}-\mathrm{CH}_{3}$ & -345.787149 & 0.124261 & 0.133330 & -345.250653 \\
\hline trans- $\mathrm{HC}_{3} \mathrm{OOC}-\mathrm{CH}=\mathrm{CH}-\mathrm{CH}_{3}$ & -345.789828 & 0.124113 & 0.133205 & -345.253448 \\
\hline cis- $\mathrm{HC}_{3} \mathrm{OOC}-\mathrm{CH}=\mathrm{CH}-\mathrm{CH}_{2}-\mathrm{CH}_{3}$ & -385.100439 & 0.153236 & 0.163538 & -384.483362 \\
\hline trans- $\mathrm{HC}_{3} \mathrm{OOC}-\mathrm{CH}=\mathrm{CH}-\mathrm{CH}_{2}-\mathrm{CH}_{3}$ & -385.103246 & 0.152903 & 0.163333 & -384.486097 \\
\hline cis-3,3-dimethylacrylic acid & -345.801195 & 0.124188 & 0.133092 & -345.272723 \\
\hline trans-3,3-dimethylacrylic acid & -345.797869 & 0.124138 & 0.133120 & -345.270074 \\
\hline cis-methyl ester 3,3-dimethylacrylic acid & -385.107738 & 0.152345 & 0.162901 & -384.490483 \\
\hline trans-methyl ester 3,3-dimethylacrylic acid & -385.104082 & 0.152379 & 0.162995 & -384.487476 \\
\hline cis-ethyl ester 3,3-dimethylacrylic acid & -424.426619 & 0.180799 & 0.192650 & -423.728131 \\
\hline trans-ethyl ester 3,3-dimethylacrylic acid & -424.422906 & 0.180797 & 0.192740 & -423.725341 \\
\hline
\end{tabular}

${ }^{\mathrm{a}}$ scaling factor 0.96

\section{Calculation of the gaseous enthalpy of formation of acrylic acid}

Experimental data on acrylic acid is in disarray according to the reference (Notario, R.; Roux, M.V.; Bors, D.A.; Liebman, J.F. 2007, 18, 395-398.).

In order to obtain the experimental value of $\Delta_{\mathrm{f}} \mathrm{H}_{\mathrm{m}}^{\circ}$ (l) for this compound we selected the following data:

$\Delta_{\mathrm{f}} \mathrm{H}_{\mathrm{m}}^{\circ}(1)=-377.8 \pm 9.3 \mathrm{~kJ} \cdot \mathrm{mol}^{-1}$ from Vilcu, R.; Perisanu, S. Rev. Roum. Chim., 1980, 25, 619-624. and

$\Delta_{\mathrm{f}} \mathrm{H}_{\mathrm{m}}^{\circ}(1)=-379.1 \pm 5.0 \mathrm{~kJ} \cdot \mathrm{mol}^{-1}$ from Moureu, C.; Boutaric, A., J. Chim. Phys., 1920, 18, 348-352.

The average value $\Delta_{\mathrm{f}} \mathrm{H}_{\mathrm{m}}^{\circ}(\mathrm{l})=\mathbf{- 3 7 8 . 5} \pm 1.4 \mathrm{~kJ} \cdot \mathrm{mol}^{-1}$

$\Delta_{1}^{\mathrm{g}} \mathrm{H}_{\mathrm{m}}(298.15 \mathrm{~K})=53.1 \pm 4.2 \mathrm{~kJ} \cdot \mathrm{mol}^{-1}$ from Van-chin-syan, Yu.Ya.; Kochubei, V.V.; Sergeev, V.V.; Raevskii, Yu.A.; Gerasimchuk, S.I.; Kotovich, Kh.Z. Sov. J. Chem. Phys. (Engl. Transl.), 1996, 70, 1789-1794. 
$\Delta_{1}^{\mathrm{g}} \mathrm{H}_{\mathrm{m}}(298.15 \mathrm{~K})=57.3 \pm 1.3 \mathrm{~kJ} \cdot \mathrm{mol}^{-1}$ from Vilcu, R.; Perisanu, S. Rev. Roum. Chim., 1980, 25, 619-624.

$\Delta_{1}^{\mathrm{g}} \mathrm{H}_{\mathrm{m}}(298.15 \mathrm{~K})=55.3 \mathrm{~kJ} \cdot \mathrm{mol}^{-1}$ from data treatment which we made using the vapor pressure measurements (293-343 K) reported by Gubkov, A.N.; Fermor, N.A.; Smirnov, N.I. Zh. Prikl. Khim. (Leningrad), 1964, 37, 2204-2210. and data on association enthalpy of acrylic acid given in Büttner, R.; Maurer, G. Ber. Bunsenges. Phys. Chem. 1983, 87, 877-882.

The average value $\Delta_{1}^{\mathrm{g}} \mathrm{H}_{\mathrm{m}}(298.15 \mathrm{~K})=\mathbf{5 5 . 2} \pm \mathbf{1 . 2} \mathbf{~ k J \cdot \mathbf { m o l } ^ { - 1 }}$

The enthalpy of formation in the gaseous state:

$\Delta_{\mathrm{f}} \mathrm{H}_{\mathrm{m}}^{\circ}(\mathrm{g})=\Delta_{\mathrm{f}} \mathrm{H}_{\mathrm{m}}^{\circ}(\mathrm{l})+\Delta_{\mathrm{l}}^{\mathrm{g}} \mathrm{H}_{\mathrm{m}}=-378.5+55.05=-323.3 \pm 1.8 \mathrm{~kJ} \cdot \mathrm{mol}^{-1}$

the latter value is in excellent agreement with the recommendation $-321 \pm 38 \mathrm{~kJ} \cdot \mathrm{mol}^{-1}$ derived from ab initio calculations (Notario, R.; Roux, M.V.; Bors, D.A.; Liebman, J.F. Struct. Chem. 2007, 18, 395-398) recently. 